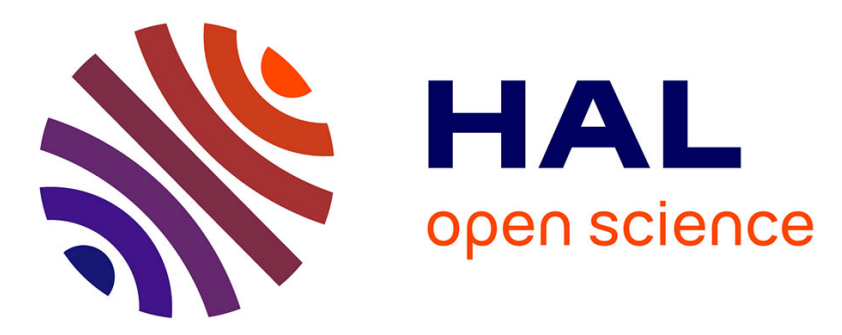

\title{
Using high-frequency vibrations and non-linear inclusions to create metamaterials with adjustable effective properties
}

\author{
B.S. Lazarov, J.J. Thomsen
}

\section{- To cite this version:}

B.S. Lazarov, J.J. Thomsen. Using high-frequency vibrations and non-linear inclusions to create metamaterials with adjustable effective properties. International Journal of Non-Linear Mechanics, 2008, 44 (1), pp.90. 10.1016/j.ijnonlinmec.2008.09.001 . hal-00487391

\section{HAL Id: hal-00487391 \\ https://hal.science/hal-00487391}

Submitted on 29 May 2010

HAL is a multi-disciplinary open access archive for the deposit and dissemination of scientific research documents, whether they are published or not. The documents may come from teaching and research institutions in France or abroad, or from public or private research centers.
L'archive ouverte pluridisciplinaire HAL, est destinée au dépôt et à la diffusion de documents scientifiques de niveau recherche, publiés ou non, émanant des établissements d'enseignement et de recherche français ou étrangers, des laboratoires publics ou privés. 


\section{Author's Accepted Manuscript}

Using high-frequency vibrations and non-linear inclusions to create metamaterials with adjustable effective properties

B.S. Lazarov, J.J. Thomsen

PII: $\quad$ S0020-7462(08)00164-9

DOI: $\quad$ doi:10.1016/j.ijnonlinmec.2008.09.001

Reference: $\quad$ NLM 1525

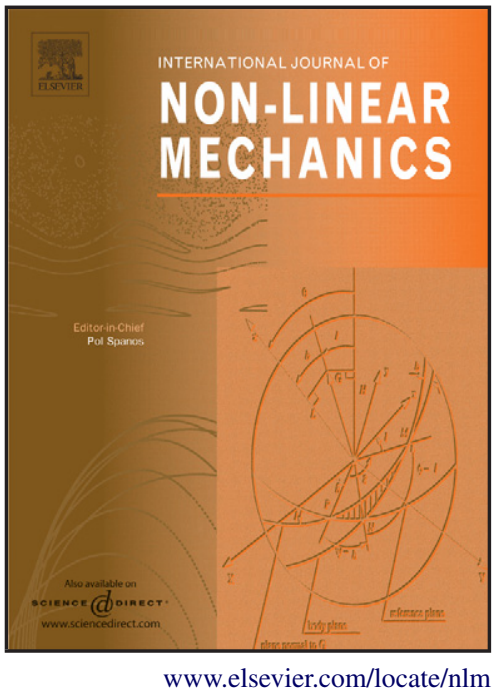

To appear in: International Journal of Non-

Linear Mechanics

Received date: 17 March 2008

Revised date: 8 September 2008

Accepted date: 18 September 2008

Cite this article as: B.S. Lazarov and J.J. Thomsen, Using high-frequency vibrations and non-linear inclusions to create metamaterials with adjustable effective properties, International Journal of Non-Linear Mechanics (2008), doi:10.1016/j.ijnonlinmec.2008.09.001

This is a PDF file of an unedited manuscript that has been accepted for publication. As a service to our customers we are providing this early version of the manuscript. The manuscript will undergo copyediting, typesetting, and review of the resulting galley proof before it is published in its final citable form. Please note that during the production process errors may be discovered which could affect the content, and all legal disclaimers that apply to the journal pertain. 


\title{
Using high-frequency vibrations and
}

\section{non-linear inclusions to create metamaterials with adjustable effective properties}

\author{
B. S. Lazarov* and J. J. Thomsen \\ Solid Mechanics, Department of Mechanical Engineering, Technical University of \\ Denmark, Nils Koppels Alle, Building 404, DK-2800 Kgs. Lyngby, Denmark
}

\begin{abstract}
We investigate how high-frequency (HF) excitation combined with strongly nonlinear elasticity may influence the effective properties for low-frequency wave propagation. The HF effects are demonstrated for linear spring-mass chains with embedded non-linear parts. The investigated mechanical systems can be viewed as a 1D model of materials with non-linear inclusions. The presented analytical and numerical results show that effective material properties can be altered by establishing HF standing waves in the non-linear regions of the chain. In addition, it is demonstrated how true static displacements and forces can be created by using HF excitation with structures having asymmetric displacement-force characteristics.
\end{abstract}

Key words: wave propagation, high-frequency excitation, stiffening, metamaterials, nonlinearity

PACS:

\footnotetext{
* Corresponding author.

Email addresses: bsl@mek.dtu.dk (B. S. Lazarov), jjt@mek.dtu.dk ( J. J.
} 


\section{Introduction}

The purpose of this article is to investigate some of the possibilities to change the effective material or structural properties for low frequency (LF) wave propagation by using high-frequency (HF) external excitation combined with strong non-linear elastic material behavior. The ability to create dynamical changes in the materials can be utilized in many problems in structural and mechanical engineering, e.g., creating elasto-mechanical filters, waveguides, ultrasonic devices, opto-mechanical components, sound and vibration isolation, health monitoring. In addition studying further the HF effects of non-linear systems can lead to an improved and safer design of mechanical systems and components.

General HF effects in non-linear systems have been studied analytically, numerically and experimentally in many scientific works, e.g., $[2-4,6,9,10,12,14,15]$. HF excitation can change various aspects of the system behavior. This work utilizes the so-called stiffening effect [4], which results in changes of the effective stiffness for low frequency excitation and the natural frequencies of the system. It can also influence the equilibrium states and their stability. A well known example of the stiffening effect is the upside-down equilibrium stabilization of the Kapitza pendulum on a vibrating support [16], which belongs to the class of parametrically excited systems. The effects of HF parametric excitation are well understood, and have been studied analytically and numerically in many articles $[12,6,4]$. Experimental verification for various physical systems is presented in $[13,20,3,5]$. Stiffening can also be observed in externally excited non-linear systems. Analytical and numerical results for calculating the

$\overline{\text { Thomsen). }}$ 
averaged motion of an externally excited system are presented in [8]. A major part of the references cited above addresses single degree of freedom systems. HF effects for a general class of multi degree of freedom (MDOF) systems have been studied in $[2,4]$, and later for continuous systems in [3].

It has been suggested in recent works $[2,17,7]$ to use modulation of the structural/material characteristics in space and time, in order to create materials and structures with adjustable effective properties. Such materials are known as dynamic materials $[2,17]$. In $[19,18]$ HF parametric excitation has been applied to control the effective material properties. In [7] by assuming the existence of a HF standing wave in a continuous non-linear systems, the effective material properties for the LF motion (slow motion) are derived analytically. Compared to the approaches in $[19,18]$, where distributed actuation and control are used, the advantage of the approach in [7] is the use of a single excitation source for generating the HF field. However, in contrast to the parametric case, where the stiffness change depends on the frequency of the excitation, for the externally excited non-linear system it does not depend on the frequency of the excitation, and is proportional to the response amplitude and the strength of the non-linearity. If the non-linearities are cubic, the change in the effective material stiffness is predicted to be proportional to the square of the HF response amplitude. The same effect for undamped Fermi-Pasta-Ulam chains has also been predicted analytically in [24].

The stiffening can be amplified by increasing the amplitude of the HF standing wave and/or by increasing the strength of the non-linearity. Establishing stable finite amplitude waves in a non-linear medium is not a trivial matter in the general case. Numerical and analytical studies of non-linear chains $[21,22]$ show, that the energy level per particle necessary to start chaotic behavior 
increases with fewer particles in the chain, and thus stable standing waves are easier to establish in shorter chains. This property, combined with the fact that the waves with frequencies above a given threshold cannot propagate along linear chains [1], is used in the present work, in order to create a HF standing wave in a finite non-linear region around the location of the HF excitation. In this way some of the problems related to stability of the HF response can be avoided.

The system considered in the present study is shown in Figure 1. Short nonlinear chains shown with bold lines are embedded in a longer linear one, and the HF excitation is explicitly applied in a non-parametric way in the regions with non-linearities. The HF frequency is within the stop band of the linear chain, and the HF response is localized around the location of the applied excitation.

Various asymptotic methods have been used in the past, to analyze the HF effects in linear and non-linear systems, e.g. $[2-4,9,15]$. The Method of Direct Separation of Motion (MDSM) is utilized in this article. The method originates from the work [16]. Later it was generalized by Blekhman [2], and is utilized in $[3-5,7,8,12,13,15]$. The main assumption in MDSM is that the motion can be represented as a superposition of fast and slow oscillations. The equations for the slow motion are obtained by averaging.

The article is separated into two major parts - theoretical analysis and numerical testing. After introducing the equations of motion for the chain shown in Figure 1, the MDSM is used to obtain the equations governing the slow and fast motions. The obtained analytical results and predictions for the stiffening effect are tested later by numerical simulations. 


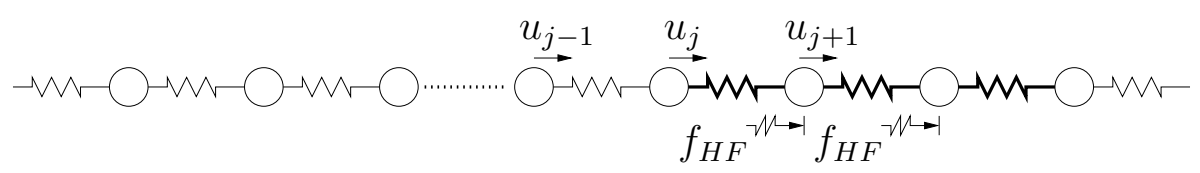

Fig. 1. Spring mass system.

\section{Mechanical model}

The considered system, shown in Figure 1, consists of oscillators with mass $m$, which are interconnected with springs. The oscillators are labeled with an index $j$, and their displacements around the static equilibrium position are denoted with $\tilde{u}_{j}$. The spring restoring forces are derived from a potential which depends only on the relative distance between the neighbor masses. The equation of motion for the $j^{\text {th }}$ mass can be written as:

$$
m \frac{\mathrm{d}^{2} \tilde{u}_{j}}{\mathrm{~d} \tilde{t}^{2}}+\tilde{c}\left(2 \frac{\mathrm{d} \tilde{u}_{j}}{\mathrm{~d} \tilde{t}}-\frac{\mathrm{d} \tilde{u}_{j-1}}{\mathrm{~d} \tilde{t}}-\frac{\mathrm{d} \tilde{u}_{j+1}}{\mathrm{~d} \tilde{t}}\right)+k \frac{\partial \tilde{V}}{\partial \tilde{u}_{j}}=\tilde{f}_{j}(t)
$$

where $\tilde{f}_{j}(t)$ is the external excitation applied to mass $j$, and $c$ is a linear viscous damping coefficient, which is assumed to be small. By introducing normalized time $t=\tilde{\omega} \tilde{t}$, where $\tilde{\omega}=\sqrt{k / m},(1)$ becomes:

$$
\frac{\mathrm{d}^{2} u_{j}}{\mathrm{~d} t^{2}}+c\left(2 \frac{\mathrm{d} u_{j}}{\mathrm{~d} t}-\frac{\mathrm{d} u_{j-1}}{\mathrm{~d} t}-\frac{\mathrm{d} u_{j+1}}{\mathrm{~d} t}\right)+\frac{\partial V}{\partial u_{j}}=f_{j}(t)
$$

For a system with $N$ masses, the equations of motion can be written in a matrix form as:

$$
\frac{\mathrm{d}^{2} \mathbf{u}}{\mathrm{d} t^{2}}+\mathbf{C} \frac{\mathrm{d} \mathbf{u}}{\mathrm{d} t}+\mathbf{\Phi}(\mathbf{u})=\mathbf{f}(t)
$$

where $\mathbf{u}(t) \in R^{N}$ is a vector with an element $j$ equal to the displacement of the $j^{\text {th }}$ mass, and $\boldsymbol{\Phi}(\mathbf{u})$ is a vector function of the displacements with elements $\Phi_{j}=\partial V(\mathbf{u}) / \partial u_{j}$. The potential $V$ is assumed to be in the form of power 
series:

$$
V=\sum_{k=1}^{m} \sum_{j=1}^{N-1} \frac{a_{k, j}}{k+1} r_{j}(\mathbf{u})^{k+1}+\text { constant }
$$

so that:

$$
\Phi_{j}=\frac{\partial V(\mathbf{u})}{\partial u_{j}}=\sum_{k=1}^{m}\left[a_{k, j-1} r_{j-1}(\mathbf{u})^{k}-a_{k, j} r_{j}(\mathbf{u})^{k}\right], j=1 \ldots N-1
$$

where $r_{j}(\mathbf{u})=u_{j+1}-u_{j}, u_{0}=0$ and $u_{N}=0$. The sum $\sum_{k=1}^{m} a_{k, j-1} r_{j-1}(\mathbf{u})^{k}$

represents the restoring force of the spring interconnecting masses $j$ and $j-1$. The external excitation $\mathbf{f}(t)$ is assumed to consist of low-frequency and zero mean high frequency components:

$$
\mathbf{f}(\omega t, \Omega t)=\mathbf{f}_{L F}(\omega t)+\mathbf{f}_{H F}(\omega t, \Omega t), \Omega>>\omega
$$

where the high frequency $\Omega$ is much larger than the low one $\omega$. In addition, the HF component is assumed to fulfill the following condition:

$$
\int_{\tau_{0}}^{\tau_{0}+2 \pi} \mathbf{f}_{H F}\left(t_{s}, \tau\right) \mathrm{d} \tau=0
$$

where $\tau=\Omega t$ and $t_{s}=\omega t$ will be called fast and slow times respectively, and $t_{s}$ is assumed independent from $\tau$ in the integration.

\section{Approximate equations governing slow and fast motions}

The focus is on the LF system response, and on how the HF excitation affects the relevant system properties. Approximate equations governing the slow motion are obtained by using the Method of Direct Separation of Motion (MDSM) [2]. The solution of (3)-(7) is assumed to be in the form:

$$
\mathbf{u}=\mathbf{z}\left(t_{s}\right)+\boldsymbol{\psi}\left(t_{s}, \tau\right)
$$


where $\mathbf{z}$ is the slow or the averaged motion of the system and $\boldsymbol{\psi}$ is a fast oscillatory function. The function $\boldsymbol{\psi}\left(t_{s}, \tau\right)$ is assumed to be periodic in $\tau$ with period $2 \pi$, and is required to have a zero fast time average:

$$
\left\langle\boldsymbol{\psi}\left(t_{s}, \tau\right)\right\rangle_{\tau}=\frac{1}{2 \pi} \int_{0}^{2 \pi} \boldsymbol{\psi}\left(t_{s}, \tau\right) \mathrm{d} \tau=0
$$

By averaging (8) and using (9), the averaged solution $\left\langle\mathbf{u}\left(t_{s}, \tau\right)\right\rangle_{\tau}$ is equal to the slow motion function $\mathbf{z}\left(t_{s}\right)$. The averaging $\langle\cdot\rangle_{\tau}$ is a linear operator, and given that (9) is fulfilled, the following relations are fulfilled as well:

$$
\left\langle\boldsymbol{\psi}\left(t_{s}, \tau\right)^{\prime}\right\rangle_{\tau}=\left\langle\boldsymbol{\psi}\left(t_{s}, \tau\right)^{\prime \prime}\right\rangle_{\tau}=\left\langle\dot{\boldsymbol{\psi}}\left(t_{s}, \tau\right)^{\prime}\right\rangle_{\tau}=0
$$

where $\{\cdot\}^{\prime}$ denotes differentiation with respect to $\tau$, and $\{\cdot\}$ denotes differentiation with respect to $t_{s}$.

By inserting (8) and (6) into the equations of motion (3), and averaging with respect to the fast time, the equations for the slow motion are obtained in the following form:

$$
\omega^{2} \ddot{\mathbf{z}}+\omega \mathbf{C} \dot{\mathbf{z}}+\langle\mathbf{\Phi}(\mathbf{z}+\boldsymbol{\psi})\rangle_{\tau}=\mathbf{f}_{L F}\left(t_{s}\right)
$$

The expression for the averaged restoring force $\langle\boldsymbol{\Phi}(\mathbf{z}+\boldsymbol{\psi})\rangle_{\tau}$ in (11) is obtained by using (5), and by expanding the result on LF and HF displacement differences:

$$
\begin{aligned}
& \left\langle\Phi_{j}\right\rangle_{\tau}=\Phi_{j}(\mathbf{z})+\left\langle\Phi_{j}(\boldsymbol{\psi})\right\rangle_{\tau}+\sum_{k=1}^{m-1} \sum_{p=k+1}^{m}\left[c_{k, p, j-1} r_{j-1}(\mathbf{z})^{k}+c_{k, p, j} r_{j}(\mathbf{z})^{k}\right] \\
& c_{k, p, j}=\left(\begin{array}{l}
p \\
k
\end{array}\right) a_{p, j}\left\langle r_{j}(\psi)^{p-k}\right\rangle_{\tau}
\end{aligned}
$$


Eq. (12) can also be written in the following form:

$$
\begin{aligned}
& \left\langle\Phi_{j}\right\rangle_{\tau}=\Phi_{j}^{e q}(\mathbf{z})+\left\langle\Phi_{j}(\boldsymbol{\psi})\right\rangle_{\tau} \\
& \Phi_{j}^{e q}(\mathbf{z})=\sum_{k=1}^{m}\left[a_{k, j-1}^{e q} r_{j-1}(\mathbf{z})^{k}+a_{k, j}^{e q} r_{j}(\mathbf{z})^{k}\right] \\
& a_{k, j}^{e q}=a_{k, j}+\sum_{p=k+1}^{m} a_{p, j}\left(\begin{array}{l}
p \\
k
\end{array}\right)\left\langle r_{j}(\boldsymbol{\psi})^{p-k}\right\rangle_{\tau}
\end{aligned}
$$

In the general case the term $\left\langle\Phi_{j}(\boldsymbol{\psi})\right\rangle_{\tau} \neq 0$ and on average the HF response correspond to additional static forces in the equations governing the slow motion. The HF motion contributes to the effective stiffness and the coefficients in front of all powers of $r_{j}(\mathbf{z})$. An important case can be distinguished when the restoring forces of the springs are odd functions with respect to the relative displacements $r_{j}$, and the differences $r_{j}(\boldsymbol{\psi})$ as functions of the fast time are odd functions with respect to a point in the interval $(\tau, \tau+2 \pi]$. In this case the average of the odd powers $\left\langle r_{j}(\boldsymbol{\psi})^{2 k+1}\right\rangle_{\tau}, k=0,1,2, \ldots$, is zero, as well as the resulting coefficients in front of the even powers of $r_{i}(\mathbf{z})$. The equivalent static force $\left\langle\Phi_{j}(\boldsymbol{\psi})\right\rangle_{\tau}$ also has a zero value. The slow motion is similar to the response of the system without $\mathrm{HF}$ excitation.

An estimate of the HF response $\boldsymbol{\psi}$ is necessary, in order to evaluate the coefficients in the equations governing the slow system motion. The equations governing the HF motion are obtained by inserting (8) into (3) and subtracting (11), and read as follows:

$$
\frac{\mathrm{d} \boldsymbol{\psi}}{\mathrm{d} t^{2}}+\mathbf{C} \frac{\mathrm{d} \boldsymbol{\psi}}{\mathrm{d} t}+\boldsymbol{\Phi}(\boldsymbol{\psi})-\langle\boldsymbol{\Phi}(\boldsymbol{\psi})\rangle_{\tau}-\mathbf{V}\left(t_{s}\right)=\mathbf{f}_{H F}\left(t_{s}, \tau\right)
$$

where $\mathbf{V}\left(t_{s}\right)$ represents the last two sums in (12). The two systems of integrodifferential equations (17) and (11) describe the motion of the original system completely. However, their solution is not easier than the original equations given by (3). Further simplifications can be achieved by making assumptions 
for the magnitude of the response. By inserting the following two expressions for the fast motion derivatives:

$$
\begin{aligned}
\frac{\mathrm{d}}{\mathrm{d} t} \boldsymbol{\psi} & =\omega \dot{\boldsymbol{\psi}}\left(t_{s}, \tau\right)+\Omega \boldsymbol{\psi}^{\prime}\left(t_{s}, \tau\right) \\
\frac{\mathrm{d}^{2}}{\mathrm{~d} t^{2}} \boldsymbol{\psi} & =\omega^{2} \ddot{\boldsymbol{\psi}}\left(t_{s}, \tau\right)+2 \Omega \omega \dot{\boldsymbol{\psi}}^{\prime}\left(t_{s}, \tau\right)+\Omega^{2} \boldsymbol{\psi}^{\prime \prime}\left(t_{s}, \tau\right)
\end{aligned}
$$

into (17), dividing by $\Omega^{2}$, and rearranging the terms, the following equation is obtained:

$$
\boldsymbol{\psi}^{\prime \prime}+\frac{1}{\Omega} \mathbf{C} \boldsymbol{\psi}^{\prime}+\frac{1}{\Omega^{2}}\left[\boldsymbol{\Phi}(\boldsymbol{\psi})-\langle\boldsymbol{\Phi}(\boldsymbol{\psi})\rangle_{\tau}\right]+\frac{\omega^{2}}{\Omega^{2}} \ddot{\boldsymbol{\psi}}+2 \frac{\omega}{\Omega} \dot{\boldsymbol{\psi}}^{\prime}+\frac{\omega}{\Omega^{2}} \mathbf{C} \dot{\psi}-\frac{1}{\Omega^{2}} \mathbf{V}=\frac{1}{\Omega^{2}} \mathbf{f}_{H F}\left(t_{s}, \tau\right)
$$

The order of the derivatives of the fast motion with respect to the slow and the fast time is assumed to be the same:

$$
O(\dot{\boldsymbol{\psi}}) \sim O\left(\boldsymbol{\psi}^{\prime}\right) \sim O\left(\dot{\boldsymbol{\psi}}^{\prime}\right) \sim O(\ddot{\boldsymbol{\psi}}) \sim O(\boldsymbol{\psi})
$$

Waves with frequency $\omega$ propagating along the chain have a relatively long wave length compared to a fast wave with frequency $\Omega$. Therefore the differences $r_{j}(\mathbf{z})$ and their powers have relatively small values, and thus the term $\mathbf{V}\left(t_{s}\right) / \Omega^{2}$ is assumed to be small. For a given response this assumption and (21) can be checked a posteriori. Using the above arguments and the initial assumption that $\Omega \gg \omega$, the following simplified system of equations for the fast motion is obtained:

$$
\boldsymbol{\psi}^{\prime \prime}++\frac{1}{\Omega} \mathbf{C} \boldsymbol{\psi}^{\prime}+\frac{1}{\Omega^{2}}\left[\boldsymbol{\Phi}(\boldsymbol{\psi})-\langle\boldsymbol{\Phi}(\boldsymbol{\psi})\rangle_{\tau}\right]+O\left(\frac{\omega}{\Omega}\right)=\frac{1}{\Omega^{2}} \mathbf{f}_{H F}\left(t_{s}, \tau\right)
$$

In the case when $\Omega^{2}$ is much larger than the stiffness-to-mass ratio of the springs interconnecting the masses, and there is a lack of resonance effects, the damping and the restoring forces in (22) can be neglected, and the so-called 
inertial approximation [2] for the HF motion is obtained:

$$
\psi^{\prime \prime}=\frac{1}{\Omega^{2}} \mathbf{f}_{H F}\left(t_{s}, \tau\right)
$$

A discussion on the applicability of the inertial approximation and a comparison with the results obtained by using (22) is given in Section 4 and is discussed more generally in [5].

\subsection{Example 1: Linear plus cubic non-linearity}

Here the expression for the restoring force for mass $j$ has the following form:

$$
\Phi_{j}^{c}(\mathbf{u})=\sum_{i=j-1}^{j} a_{1, i} r_{i}(\mathbf{u})+a_{3, i} r_{i}^{3}(\mathbf{u})
$$

The resulting mechanical model corresponds to the Fermi-Pasta-Ulam chain used in computational physics, e.g. [22]. The equivalent restoring force for the equation governing the slow motion is obtained by using (14):

$$
\begin{aligned}
& \left\langle\Phi_{j}^{c}(\mathbf{u})\right\rangle_{\tau}=\left\langle\Phi_{j}^{c}(\boldsymbol{\psi})\right\rangle_{\tau}+\sum_{i=j-1}^{j} a_{1, i}^{e q} r_{i}(\mathbf{z})+a_{3, i}^{e q} r_{i}^{3}(\mathbf{z}) \\
& a_{1, i}^{e q}=a_{1, i}+3 a_{3, i}\left\langle r_{i}^{2}(\boldsymbol{\psi})\right\rangle_{\tau} \\
& a_{3, i}^{e q}=a_{3, i}
\end{aligned}
$$

It is assumed that (9) is fulfilled, and hence the coefficients in front of the even powers of $r_{j}(\mathbf{z})$ will be zero. As it appears, for the slow motion, the HF response results in an increase or a decrease of the linear stiffness of the springs between the masses, depending on the sign of $a_{3, i}$. The change is proportional to the degree of the non-linearity and the square of the HF amplitude. Similar result for a continuous system are obtained in [7]. The high order non-linear terms in the restoring force are not affected. 


\subsection{Example 2: Essentially cubic non-linearity}

Here the coefficient $a_{1, i}$ in (24) is zero, and the equivalent coefficient (26) becomes:

$$
a_{1, i}^{e q}=3 a_{3, i}\left\langle r_{i}^{2}(\boldsymbol{\psi})\right\rangle_{\tau}
$$

The HF response in this case results in a significant change of the system behavior. Slow waves with small amplitudes will propagate along the HF excited chain in a similar way as in a linear chain with spring stiffness given by (28).

\subsection{Example 3: Asymmetric non-linearity}

Here the restoring force compared to (24) is extended with an even order non-linear term and an additional fifth order term.

$$
\Phi_{j}(\mathbf{u})=\sum_{i=j-1}^{j} a_{1, i} r_{i}(\mathbf{u})+a_{2, i} r_{i}^{2}(\mathbf{u})+a_{3, i} r_{i}^{3}(\mathbf{u})+a_{5, i} r_{i}^{5}(\mathbf{u})
$$

The equivalent coefficients in (15) become as follows:

$$
\begin{aligned}
a_{1, i}^{e q} & =a_{1, i}+3 a_{3, i}\left\langle r_{i}^{2}(\boldsymbol{\psi})\right\rangle_{\tau}+5 a_{5, i}\left\langle r_{i}^{4}(\boldsymbol{\psi})\right\rangle_{\tau} \\
a_{2, i}^{e q} & =a_{2, j}+10 a_{5, j}\left\langle r_{i}^{3}(\boldsymbol{\psi})\right\rangle_{\tau} \\
a_{3, i}^{e q} & =a_{3, i}+10 a_{5, i}\left\langle r_{i}^{2}(\boldsymbol{\psi})\right\rangle_{\tau}
\end{aligned}
$$

The stiffening effect compared to Example 1 is stronger for a HF response with the same amplitude, due to the additional positive term in (31). In addition, due to the even power in the restoring force, the average $\langle\boldsymbol{\Psi}(\boldsymbol{\psi})\rangle \neq \mathbf{0}$ and the $\mathrm{HF}$ motion will result in an additional equivalent static force for the slow motion. 


\subsection{Analytical predictions - summary}

The analytical derivations show, that explicitly applied HF external excitation causes changes in the effective stiffness for a LF wave propagation. The stiffness change is proportional to the amplitude of the relative displacements and to the strength of the nonlinearity. In chains with springs having essentially nonlinear behavior, the HF excitation adds effective linear stiffness for the LF motion. The HF effects in nonlinear chains rely on the establishment of a stable HF periodic motion with a large amplitude of the relative displacements.

\section{Numerical simulations and examples}

Numerical simulations are performed with a finite spring-mass chain, using standard numerical integration routines from the SUNDIALS library [23], to test the above analytical predictions. Alteration of the chain stiffness leads to a change of the wave velocity along it. The stiffening effect can be observed by registration of these changes for a system with applied HF excitation. The slow motion has a relatively large wave length, and the contribution of the highorder displacement differences to the restoring force can be neglected. Thus, the wave propagation in the chain with a HF excitation can be compared with the wave propagation in an equivalent linear system with parameters estimated by using (16). For shorter waves with large amplitude it might be necessary to take into account the high order terms in the equivalent restoring force for the slow motion, however these cases are outside of the scope of this article. The numerical tests are performed for stiffening non-linearity. In the case of a softening, a decrease of the wave velocity can be observed. Depending 
on the HF response amplitude and the type of the softening non-linearity, the HF excitation may also result in destabilization of the equilibrium position.

The simplest model to illustrate the predicted effects, from the numerical point of view, would be a chain with repeatable non-linear springs and masses. Such a system possesses rich dynamic behavior with multiple static and dynamic equilibrium positions. The system response can easily approach chaotic regime. It is demonstrated in $[22,21]$, that the energy per mass, necessary for obtaining chaotic behavior, is larger for shorter chains, i.e., for shorter chains a larger relative displacement amplitude is necessary, to observe chaotic motion. Therefore, high energy stable oscillatory response can be established by localizing the HF response in short non-linear parts of the chain. Waves can propagate unattenuated along linear chains within specific bands of frequencies called propagation or pass bands, and attenuate within bands of frequencies called stop bands, attenuation zones or band gaps [1]. By embedding the short nonlinear chain in a longer linear one and applying HF excitation with a frequency within the stop bands of the linear chain, the HF response can be localized in the non-linear inclusions.

The investigated system is a chain with 2000 masses. At both ends the displacements are set to be zero. In every cell of twenty springs and twenty masses, one pair of neighboring springs is elastically nonlinear, while the other springs are linear (Figure 2). The HF response is generated by harmonic excitation with amplitude raising slowly from zero to the final value $A_{H F}$.

$$
f_{H F}=(1-\exp (t / C)) A_{H F} \sin (\Omega t)
$$

Initially, large damping is introduced in the system, as dashpots between the 


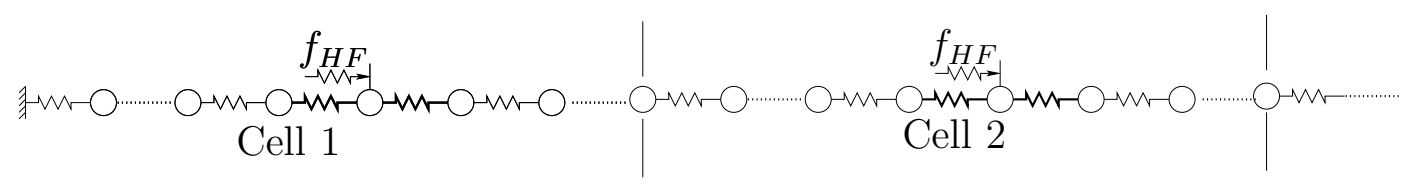

Fig. 2. Part of spring mass system with non-linear inclusions.

masses, and the damping coefficients decrease slowly to a prescribed small value with the same rate as the $\mathrm{HF}$ amplitude. In this way the transient $\mathrm{HF}$ response is damped faster, and the contribution of the damping forces to the dynamic equilibrium, when a steady state is reached, is small. The fast decay of the transients helps the system to reach a steady state oscillatory motion, rather than a complex quasiperiodic or chaotic response. Once a steady state due to the HF excitation is reached, a Gaussian modulated pulse is generated by prescribing the displacement at the left end of the chain:

$$
u_{0}(t)=\exp \left(-\alpha\left(t-t_{g}\right)\right) A_{w} \cos \left(\omega t-t_{g}\right)
$$

where $t_{g}=3500+3 T, T=2 \pi / \omega, A_{w}$ is the pulse amplitude, and $\omega$ is the slow frequency. The wave velocity for the slow motion can be estimated by finding the displacement maximum $\left\langle u_{j}\right\rangle^{\max }$ and $\left\langle u_{i}\right\rangle^{\text {max }}$, and by using the following expression:

$$
v_{w}=\frac{j-i}{t_{j}-t_{i}}
$$

where $t_{j}$ and $t_{i}$ are the time instances, when mass $j$ and mass $i$ reach the maximum value of the displacements. For the equivalent linear undamped system the group wave velocity can be calculated as a derivative of the dispersion relation $\nu(\omega)$ with respect to $\omega$, where $\nu$ is the phase velocity of a harmonic wave with frequency $\omega[1]$. 


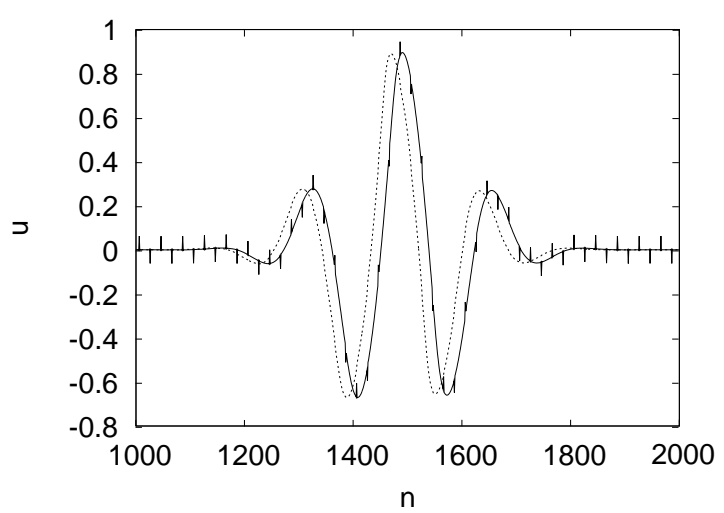

Fig. 3. Plots of the displacement response for part of a chain with 2000 masses at time $t=6400.4$ with applied HF excitation (solid line) and without HF excitation (dashed line).

\subsection{Inclusions with linear plus cubic non-linear behavior}

The stiffening effect is illustrated in Figure 3. Snapshots of the spatial displacements of two chains, with and without HF excitation, are presented. A Gaussian modulated pulse with frequency $\omega=0.025$ is generated by using (34) in both chains. The pulse in the chain with the HF excitation propagates faster than the one in a chain without it. The small spikes in the system with HF excitation are due to the localized HF response in the non-linear inclusions.

A plot of the wave velocity versus the amplitude of the HF excitation, for a chain with non-linear inclusions with restoring force given by (24), is shown in Figure 4. An increase of the velocity, and hence, an increase of the equivalent stiffness is observed, which is in line with the analytical prediction. The velocity obtained by numerical simulations for the non-linear system coincides very well with the one obtained from numerical simulations and analytical calculations for the equivalent linear system. The equivalent parameters are estimated by using (26) with HF response obtained from the numerical simu- 


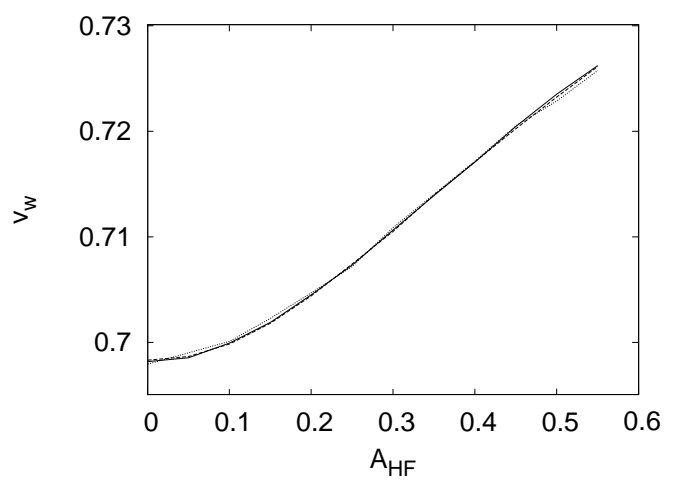

Fig. 4. Plots of the group wave velocity for a pulse with a base frequency $\omega=0.025$ in a chain with two non-linear inclusions on every 20 springs, and HF forces (amplitude $A_{H F}$, frequency $\Omega=\pi$ ) applied on the masses between the neighbor non-linear springs. The linear springs have stiffness $a_{0}=0.5$, and the non-linear springs restoring forces have parameters $a_{1}=0.4$ and $a_{3}=0.4 \gamma$, where $\gamma=200$. The solid line is obtained by numerical simulations with the non-linear inclusions, the dashed line by numerical simulations with the equivalent linear system obtained by replacement of the non-linear springs with equivalent linear ones. The dotted line is obtained for the equivalent system by using analytical prediction for the undamped system.

lations.

If the restoring and damping forces in (22) can be considered to be small compared to the inertial force, the inertial approximation (23) for the HF motion is obtained. An estimate of the wave velocity by using the inertial approximation and numerical simulation with the non-linear system, is shown in Figure 5. As the HF frequency increases, the inertial approximation gives better results. For lower frequencies the estimation starts to deviate from the numerical simulations when the amplitude of the excitation increases. Increasing the amplitude of the excitation is leading to an increase of the amplitude of the HF response, and thus, the restoring force term in (23) is having large contribution to the force balance. Therefore, for highly non-linear 


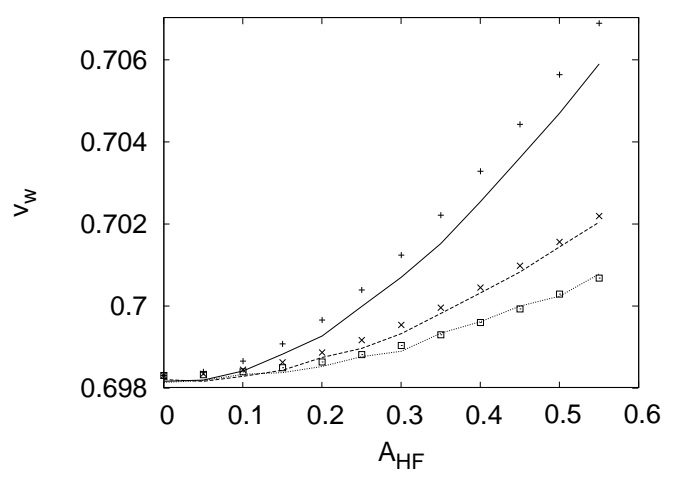

Fig. 5. Comparison of the wave speed obtained by using inertial approximation and numerical simulations for the same system as in Figure 4, and HF excitation with frequency $\Omega=1.5 \pi, 1.8 \pi, 2 \pi$ shown with solid, dashed and dotted lines respectively. The values obtained by the inertial approximation are shown with point markers. systems, the inertial approximation will not lead to a good prediction, even in the case when resonance effects are avoided. For weakly non-linear systems it leads to very good results.

The wave speed in chains with inclusions having different strengths of the non-linearity is shown in Figure 6. As the inertial term is dominant in the equations of motion governing the HF motion, the increase of the excitation amplitude leads to an increase of the amplitude of the HF motion, therefore, stronger non-linearity will lead to a higher equivalent stiffness and a higher wave speed, as shown in Figure 6.

\subsection{Inclusions with essential non-linearity}

HF excitation in combination with strong non-linearity can lead to qualitative changes. If the inclusions in the chain are essentially non-linear $\left(a_{1}=0\right)$, it is observed that a wave propagating along the chain will approach a shock wave after passing through a few non-linear inclusions. If a HF excitation is applied, 

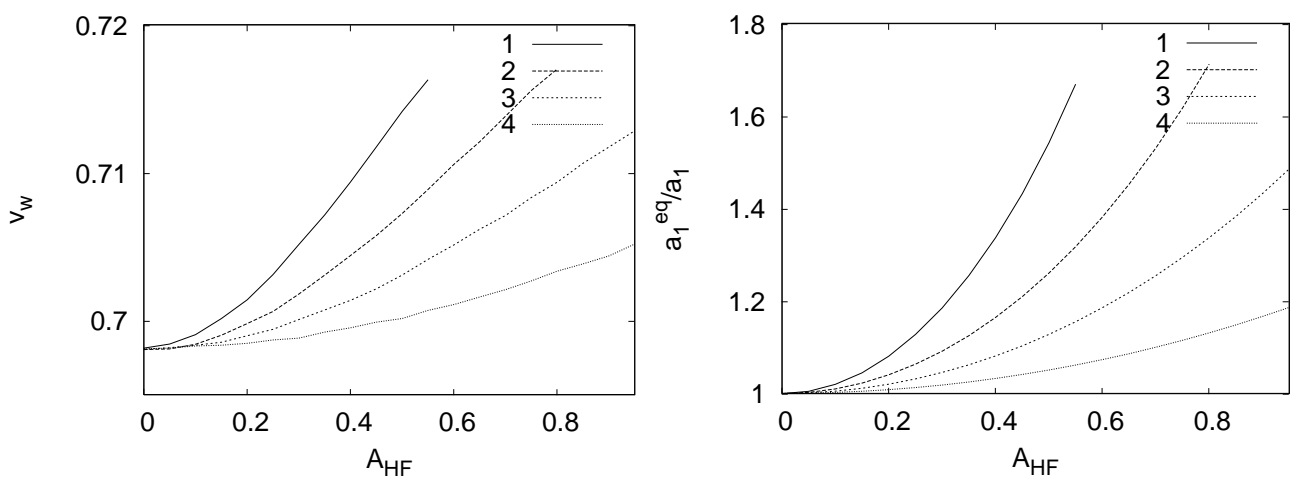

Fig. 6. The left plot shows the group wave velocity for pulse with base frequency $\omega=0.025$ obtained by numerical simulation for the same system as in Figure 4 with HF excitation frequency $\Omega=\pi$, and $\gamma=100,50,25,10$ for plots $1,2,3$ and 4 . The second plot shows the equivalent stiffness increase of the non-linear spring with respect to the amplitude of the excitation $A_{H F}$.

the HF motion generates an equivalent linear stiffness, as shown by (28), and the chain becomes transparent for long waves. Comparison of the wave velocity for an equivalent linear system and numerical simulations with the non-linear system, is shown in Figure 7. The amplitude of the generated long wave in this case is $A_{w}=0.1$. The system response does not show any unstable behavior in the considered time/space interval, and the results obtained by using the equivalent linear system coincide very well with those obtained by numerical simulations for the non-linear system. The equivalent stiffness in this case can be significantly altered due to the strong non-linearity.

\subsection{Inclusions with high-order non-linearities}

The results presented in Section 3 and derived with the polynomial form of the potential are valid for a much wider class of systems. The equations (4) and (5) can be viewed as a Taylor expansion of the actual potential or the 

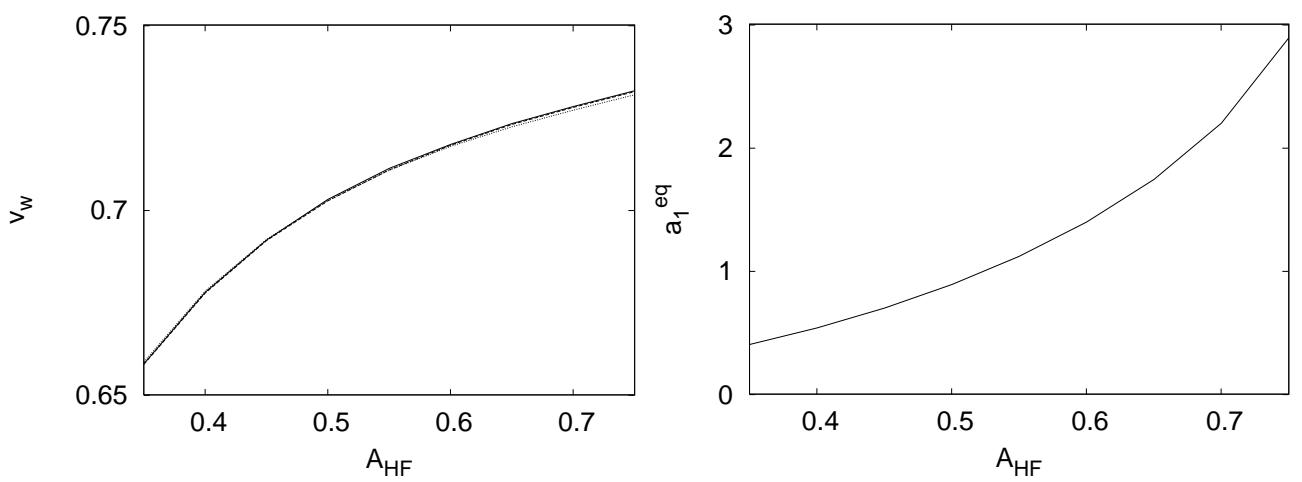

Fig. 7. The left plot shows the group wave velocity for a pulse with base frequency $\omega=0.025$ in a chain with two essentially non-linear inclusions on every 20 springs and HF force (amplitude $A_{H F}$, frequency $\Omega=\pi$ ) applied on the mass between the two non-linear springs. The non-linear springs have the following parameters $a_{1}=0.0$ and $a_{3}=0.5 \gamma$, where $\gamma=200$. The solid line is obtained by numerical simulations with the non-linear system, the dashed line - by numerical simulations with the equivalent linear system, and the dotted line - by analytical prediction for the undamped system. The second plot shows the equivalent linear stiffness as a function of the excitation amplitude.

restoring force. The wave velocity versus the amplitude of the HF excitation for a system with 2 non-linear inclusions on every twenty springs are shown in Figure 8. The restoring forces of the non-linear springs for the three presented cases are given as:

$$
\begin{aligned}
& f_{p}=\frac{a_{1}}{2 n}\left(\frac{1}{(1-r(u))^{n}}-\frac{1}{(1+r(u))^{n}}\right) \\
& f_{e}=\frac{a_{1}}{2 \beta}\left(\left(1-e^{-\beta r(u)}\right) e^{-\beta r(u)}-\left(1-e^{\beta r(u)}\right) e^{\beta r(u)}\right) \\
& f_{c}=a_{1} r(u)+a_{1} \frac{7 \beta^{2}}{6} r(u)^{3}
\end{aligned}
$$

The values of the parameters $\beta$ and $n$ are chosen so that the coefficients in front of the third power in the Taylor expansion are equal. The expression for the cubic restoring force (38) is equal to the first two terms of the expansion into 
Taylor series of (37). For small amplitudes of the HF response the equivalent wave speed in the chain is almost the same for all three restoring forces. As the HF amplitude increases, the high order averaged terms start to have a higher contribution to the equivalent coefficients, e.g. (30), and the equivalent system becomes stiffer for stronger non-linearity.

Although the results demonstrated so far are for short non-linear embedded chains consisting of two non-linear springs, the analytical predictions in Section 3 are valid for longer non-linear inclusions, as long as the HF motion is periodic or quasiperiodic, and the condition given by (9) is fulfilled. The wave velocity versus the amplitude of the HF excitation in a chain with four and five elements in the non-liner inclusion on every twenty springs is shown in Figure 9. The non-linear restoring force of the spring is modeled by using (37) with $\beta=\sqrt{66}$, and the equivalent linear stiffness is estimated by using (38) and (26). The fast time average is estimated by numerical simulations. The HF forces are applied to all masses in the non-linear inclusion with alternating signs, in order to create higher displacement differences.

\subsection{Generating a static force by means of $H F$ vibrations}

According to the analytical predictions in Section 3, a HF response combined with elastic non-linearity can generate an equivalent static force, if the periodic motion is not an odd function with respect to any point in the time interval $(\tau, \tau+2 \pi]$, or if the spring restoring forces are asymmetric with respect to their static equilibrium. The solution to (3), when the external excitation is a function of the fast time $\tau$ only, is sought in the form $\mathbf{u}(t)=\mathbf{z}+\boldsymbol{\psi}(\tau)$. For a prescribed HF response, which fulfills the condition given by (9), the new, 

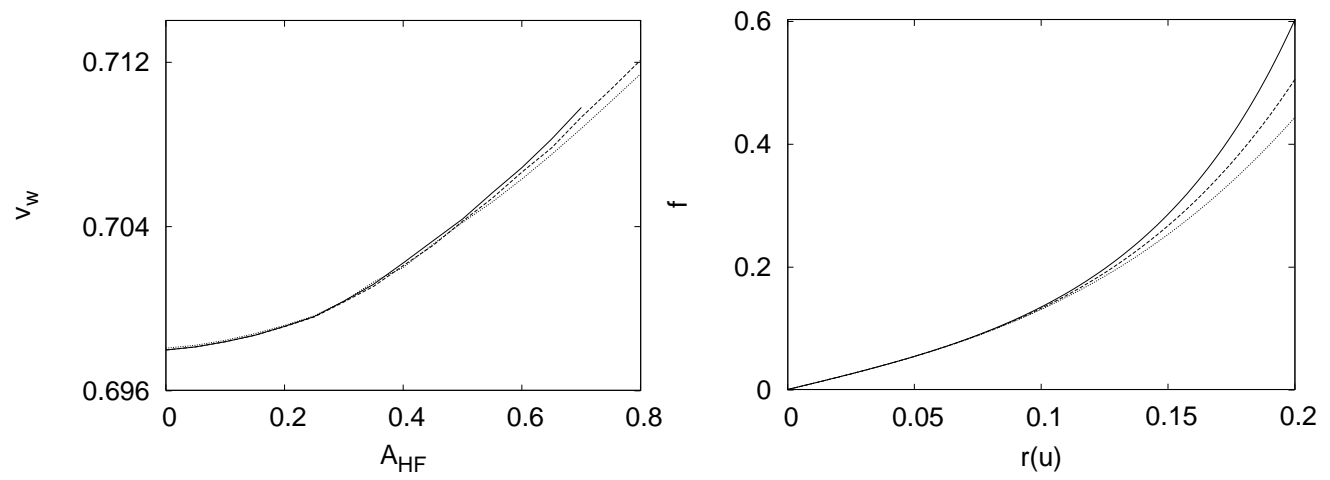

Fig. 8. Plots of the group wave velocity for a pulse with a base frequency $\omega=0.025$ in a chain with two non-linear inclusions on every 20 springs and a HF force (amplitude $A_{H F}$, frequency $\Omega=\pi$ ) applied on the mass between two non-linear springs. The restoring force $f$ of the non-linear springs is shown in the second plot as a function of the relative displacements $r(u)$. Solid, dashed and dotted lines correspond to the restoring forces given by equations (36), (37) and (38) respectively, with parameters $n=12$ and $\beta=\sqrt{26}$
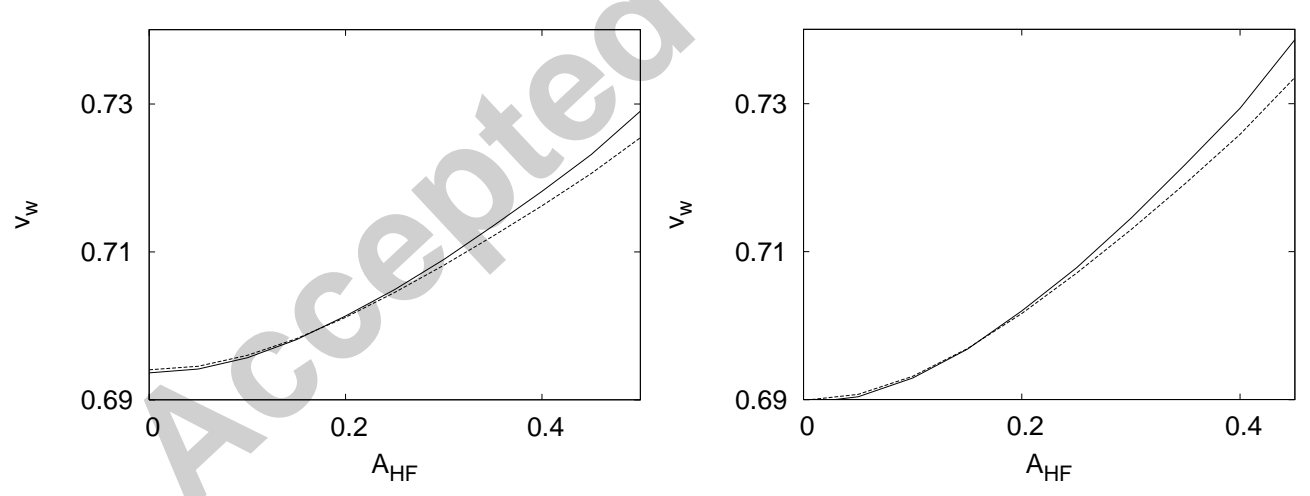

Fig. 9. Plots of the group wave velocity for a pulse with base frequency $\omega=0.025$, in a chain with 3 (left) and 4(right) non-linear inclusions on every 20 springs, and HF force (amplitude $A_{H F}$, frequency $\Omega=\pi$ ) applied to all masses between the non-linear springs. The solid line shows the results obtained by numerical simulations and the dashed line - the wave speed calculated analytically for the equivalent linear system. 


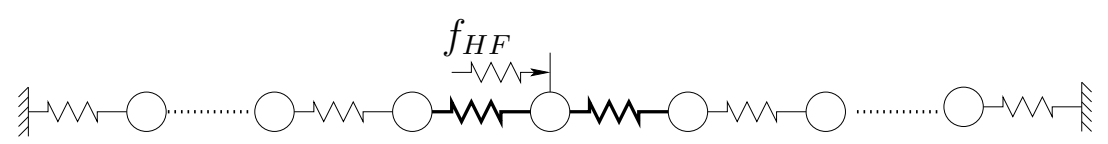

Fig. 10. Spring mass system used for generating static force by means of HF excitation.

equivalent static equilibrium position can be obtained by solving the following algebraic equation for $\mathbf{z}$.

$$
\Psi^{e q}(\mathbf{z})+\langle\Psi(\boldsymbol{\Psi})\rangle_{\tau}=0
$$

The possibility to generate an equivalent static force and a new equivalent static equilibrium position, is verified here by using numerical simulations. The investigated chain, shown in Figure 10, consists of two linear parts with 9 springs and in the middle, 2 non-linear springs shown in bold. The central mass is excited by a harmonic HF force with a frequency in the stop band of the chain, i.e., above the highest linear natural frequency. The stationary HF response is localized around the location of the HF excitation, and the response of the masses far from the excitation is non oscillatory. Therefore, the new equilibrium is a true static equilibrium, and zero mean HF oscillatory motion can be used for generating true static forces and displacements. It should be pointed out that in the case of sub-harmonic resonance, the external HF excitation may generate response within the pass band of the chain, and then the new equilibrium will be static only in the average sense.

The restoring force for the non-linear springs is derived from the widely used in particle simulations Morse potential (e.g. [21]):

$$
V_{M}(r)=\frac{a_{1}}{2 \beta^{2}}\left(1-\mathrm{e}^{-\beta r}\right)^{2}
$$


with corresponding restoring force

$$
f_{M}(r)=\frac{\mathrm{d} V_{M}(r)}{\mathrm{d} r}=\frac{a_{1}}{\beta} \mathrm{e}^{-\beta r}\left(1-\mathrm{e}^{-\beta r}\right)
$$

The strength of the non-linearity is controlled by the parameter $\beta$, and the term $1 / 2 \beta^{2}$ scales (40), so that the slope at $r=0$ of the restoring spring

force $f_{M}(r)$ is $a_{1}$. The numerical simulations are performed with excitation frequency $\pi$ and a restoring force for the non-linear springs given by (41). The resulting pre-stressing force in the linear part of the system is compared to the one obtained from the solution of (39) with equivalent parameters evaluated for the Taylor expansion of (41) given as:

$$
f_{M}(r) \approx a_{1}\left[r-\frac{3 \beta}{2} r^{2}+\frac{7 \beta^{2}}{6} r^{3}-\frac{5 \beta^{3}}{8} r^{4}+\frac{31 \beta^{4}}{120} r^{5}\right]+O\left(r^{6}\right)
$$

The time averaged powers of the HF response $\left\langle\psi^{n}\right\rangle_{\tau}$ are estimated from the numerical integration. The results are shown in the first plot in Figure 11. The amplitude of the HF response is governed mainly by the inertial term for HF excitation. The asymmetry in the force-displacement diagram is having relatively low influence on the amplitude of the HF motion. The pre-stressing force depends mainly on the fast time average $\langle\boldsymbol{\Psi}(\boldsymbol{\psi})\rangle_{\tau}$, and it is decreasing with the parameter $\beta$, as the major contribution to the fast time average comes from the even powers of the relative displacements $r$. This can be seen clearly in the second plot in Figure 11, where the three lines show the results for $\beta=2.5,5.0,10.0$. 

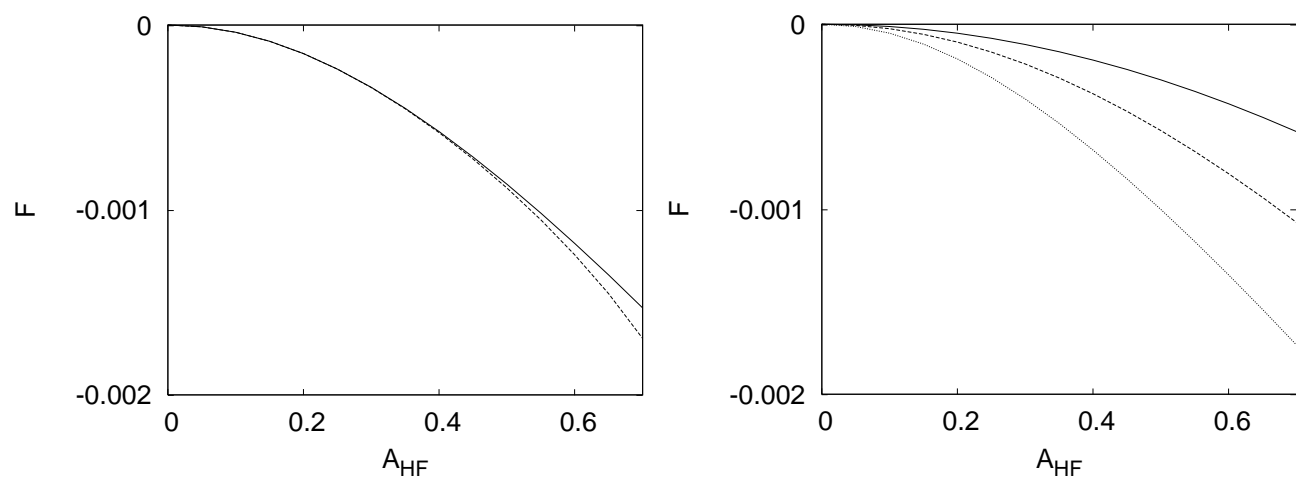

Fig. 11. Plots of the pre-stressing force in the linear part of the chain, shown in Figure 10, excited by HF harmonic force with amplitude $A_{H F}$ and frequency $\pi$. The solid line shows the force obtained from numerical integration, and the dashed line the force estimated by solving (39) with equivalent coefficients estimated by using (42) and $\beta=\sqrt{66}$. The second plot shows the pre-stressing force for $\beta=2.5,5,10$ with solid, dashed and dotted lines respectively.

\section{Conclusions}

Some possibilities of using HF excitation to alter the LF properties of 1D mechanical systems have been demonstrated. It is shown analytically and numerically that for chains with nonlinearities, non-parametric HF periodic motion causes a change of the effective stiffness and the wave speed for LF wave propagation. The change is proportional to the amplitude of the relative displacements and the strength of the non-linearity. A stable periodic motion with a large amplitude of the relative displacements is established by localizing the HF response only in parts of the chain with non-linear behavior. The localization is achieved by embedding short non-linear chains into a linear one, and by using HF excitations with frequency within the stop band of the linear chain. It has also been demonstrated that there is a possibility to create true static displacements and forces by means of HF excitation, when the 
excitation frequency is above the pass band of the discrete structure, and the restoring non-linear spring force is asymmetric. The idea can be applied to continuous structures by creating a stop-band structure around the non-linear inclusion, which acts as a HF filer.

Compared to the original idea [7] for a continuous nonlinear rod, where a single source of the HF excitation is used, here multiple excitation sources are needed, to control the effective material properties. Investigations of the stability properties of the HF response in the nonlinear inclusions, for particular types of nonlinearities, are necessary, in order to utilize the stiffening effect into practical applications. The HF response depends on the HF force amplitude, therefore, resonance effects can also be exploited, in order to minimize the need for a strong HF excitation. The energy required to change the effective material properties depends on the chosen control strategy, and optimal control solutions will be presented in subsequent articles. The presented effects for a $1 \mathrm{D}$ chain with non-linear inclusions and HF excitation can be used as a base for developing more complex one, two and three dimensional material models. The possibility to change the stiffness by a HF excitation in parts of the chain can also be used in the design of adjustable band-gap filters for a LF wave propagation.

\section{Acknowledgment}

This work was supported by grant 274-05-0498 from the Danish Research Council for Technology and Production Sciences. The authors wish to thank Professor Jakob Soendergaard Jensen for his suggestions and valuable discussions. 


\section{References}

[1] L. Brillouin, Wave propagation in periodic structures, Dover Publications Inc., 1953

[2] I.I. Blekhman, Vibrational Mechanics - Nonlinear Dynamic Effects, General Approach, Applications, World Scientific, Singapore, 2000

[3] J.J. Thomsen, Theories and experiments on the stiffening effect of highfrequency excitation for continuous elastic systems, Journal of Sound and Vibration, 260:117-139, 2003

[4] J.J. Thomsen, Some general effects of strong high-frequency excitation: Stiffening, biasing and smoothening, Journal of Sound and Vibration, 253:807831,2002

[5] J.J. Thomsen, Effective properties of mechanical systems under high-frequency excitation at multiple frequencies, Journal of Sound and Vibration, 311:12491270,2008

[6] J.J. Thomsen, Using fast vibrations to quench friction-induced oscillations, Journal of Sound and Vibration, 228:1079-1102, 1999

[7] J.J. Thomsen and I.I. Blekhman, Using nonlinearity and spatiotemporal property modulation to control effective structural properties: Dynamic rods, In Proc, of ECCOMAS Thematic Conference on Computational Methods, COMPDYN2007, Crete, Greece, 13-16 June, 2007

[8] J.J. Thomsen, Using strong non-linearity and high-frequency vibrations to control effective mechanical stiffness, In Proc. of ENOC-2008, Saint Petersburg, Russia, 2008

[9] D. Tcherniak and J.J. Thomsen, Slow effects of fast harmonic excitation for elastic structures, Nonlinear Dynamics, 17:227-246, 1998 
[10] A. Stephenson, On new type of dynamic stability, Memoirs and Proceedings of the Manchester Literary and Philosophical Society, 52:1-10, 1908

[11] S.V. Chelomei, Dynamic stability upon high-frequency parametric excitation, Soviet Physics - Doklady, 26:390-392, 1981

[12] J.S. Jensen, Buckling of an elastic beam with added high-frequency excitation, International Journal of Non-Linear Mechanics, 35:217-227, 2000

[13] J.S. Jensen, D.M. Tcherniak and J.J. Thomsen, Stiffening effects of highfrequency excitation: experiments for an axially loaded beam, Transactions of the ASME, Journal of Applied Mechanics, 67:397-402, 2000

[14] A. Fidlin, On asymptotic properties of systems with strong and very strong high-frequency excitation, Journal of Sound and Vibration, 235:219-233, 2000

[15] A. Fidlin, Nonlinear Oscillations in Mechanical Engineering, Springer-Verlag Berlin, 2005

[16] P.L. Kapitza, Dynamic stability of a pendulum with an oscillating point of suspension (in Russian), Zurnal Eksperimental'noj i Teoreticeskoj Fiziki, 21:588-597, 1951

[17] K.A. Lurie, An Introduction to the Mathematical Theory of Dynamic Materials, Springer, New York, 2007

[18] S.V. Sorokin, S.V. Grishina and O.A. Ershova, Analysis and control of vibrations of honeycomb plates by parametric stiffness modulations, Smart Materials and Structures, 10:1031-1045, 2001

[19] K.A. Lurie, Low frequency longitudinal vibrations of an elastic bar made of a dynamic material and excited at one end, Journal of Mathematical Analysis and Applications, 251:364-375, 2000 
[20] H. Yabuno and K. Tsumoto, Experimental investigation of a buckled beam under high-frequency excitation, Archive of Applied Mechanics, 77:339-351, 2007

[21] M. Rodriguez-Achahc and G. Perez, Generalized instability of periodic traveling waves in aharmonic monatomic chains, Physics Letters A, 233:383390,1997

[22] N. Budinsky and T. Bountis, Stability of nonlinear modes and chaotic properties of 1D Fermi-Pasta-Ulam lattices, Physica D, 8:445-452, 1983

[23] A.C. Hindmarsh, P.N. Brown, K.E. Grant, S.L. Lee, R. Serban, D.E. Shumaker and C.S. Woodward, SUNDIALS, suite of nonlinear and differential/algebraic equation solvers, ACM Transactions on Mathematical Software, 31:363-396, 2005

[24] Yu.A. Kosevich, Nonlinear sinusoidal waves and their superposition in anharmonic lattices, Phys. Rev. Lett., 71:2058 - 2061, 1993 